Original Research Paper

\title{
Computing Amplify-and-Forward Relay Amplification Factor to Improve Total Capacity at Destination
}

\author{
Olalekan Bello, Hushairi Zen, AL-Khalid Othman and Khairuddin Abdul Hamid \\ Department of Electrical and Electronics Engineering, University of Malaysia, Sarawak, Malaysia
}

\author{
Article history \\ Received: 29-07-2015 \\ Revised: $13-08-2015$ \\ Accepted: 03-09-2015 \\ Corresponding Author: \\ Olalekan Bello \\ Department of Electrical and \\ Electronics Engineering, \\ University of Malaysia, \\ Sarawak, Malaysia \\ Email: lekkibel@yahoo.com
}

\begin{abstract}
In this study, we propose a new Amplify-and-Forward (AF) amplification factor to improve the error performances of the current $\mathrm{AF}$ schemes and thus enhance the total capacity at the destination terminal. The proposed scheme has two degrees of freedom, meaning that it contains two variable parameters that can simultaneously vary the amplification factor instead of one as in traditional variable-gain or semi-blind relaying. The numerical results show that the proposed AF scheme outperforms some current ones with which it is compared in terms of average capacity and probability of outage and thus brings AF relaying performances closer to that of Decode-and Forward (DF) strategy which hitherto outperforms it. The results also show that by increasing or decreasing the power share of the relay, the optimal location of the relay is not only moved closer to or farther away from the destination, the total average capacity at that location is also correspondingly increased or decreased.
\end{abstract}

Keywords: Amplify-and-Forward Relays, Amplification Factor, SNR

\section{Introduction}

Relaying has been considered a promising solution in extending service coverage, especially where multiple antennas are not deployable at the terminal. Some of the most popular relaying strategies proposed in literature to provide efficient relaying include Amplify-and-Forward (AF) and Decode-and-Forward (DF) in (Laneman et al., 2004; Souryal, 2006). Saleh et al. (2009) compared the DF and AF relaying schemes with Long-Term Evolution (LTE) parameters and their findings show that DF outperforms AF in terms of spectral efficiency; however, in terms of complexity of implementation AF is considered better.

So far, the AF amplification factors that have been proposed in literature are the fixed-gain (semi blind) relay in (Laneman et al., 2004) and variable-gain relays in (Nabar et al., 2004; Anghel and Kaveh, 2003; Liu et al., 2008). The fixed-gain applies a constant gain on the received signal from the source regardless of the amplitude on the source-relay hop (Hasna and Aloini, 2004). The variable-gain amplification factors require the relay to have knowledge of instantaneous channel gains to continuously adjust their gains depending on the instantaneous channel response. However, when the Channel State Information (CSI) of the instantaneous channel coefficients are not available at the relay the second order statistic (variance) of the channel can be used to provide semi-blind relaying (Alvi and Wyne, 2014). The relay amplification factor in the AF relaying plays a major role in the resource allocation to the active users in the Orthogonal Frequency Division Multiplexing (OFDM) relaying system (Rasouli, 2012). But Berger and Wittneben (2005) had earlier noted that the way a relay factor is computed aids it in providing distributed spatial multiplexing gains that allow simultaneous access to shared network resources.

However, most recent works in (Peppas et al., 2010; Xia et al., 2011; Osamah and Michel, 2012; Cheng and Chen, 2015; Cheng and Huang, 2015) have only concentrated on investigating either the fixed, semi-blind and variable-gain $\mathrm{AF}$ systems in terms of outage probability, average bit/symbol error rate and ergodic capacity for various relay transmission and fading channel models and no serious attentions have been given to how to re-compute the existing amplification factors to generally improve the performances of AF relaying.

The main function of the amplification factor is ideally to equalize the effect of the channel fading between the source and the relay and to achieve that it uses a variable channel gain parameter, i.e., channel variance or channel coefficient in its denominator; while in its numerator some fixed scaling constant, which can further affect amplification, is specified. This type of amplification factor can be said to possess one degree of freedom, because it uses only one variable parameter. 
However, in the process of equalizing the effect of channel fading the AF schemes also inadvertently amplify the effective noise term thus limiting the overall system performances of $\mathrm{AF}$ compared to that of $\mathrm{DF}$ relaying. Therefore, in order to minimize this combined effect of fading and noise, we propose a new amplification factor which contains two degrees of freedom rather than one as in the current $\mathrm{AF}$ ones.

The numerical results show that the proposed $\mathrm{AF}$ scheme achieves a higher average capacity and lower outage probability compared to current ones. The rest of this paper is organized as follows. In section 2, we establish a system model and relay transmission. In section 3, we derive a new amplification factor. In section 4 , we present numerical results. In section 5 , we summarize our work.

\section{System Model and Relay Transmission}

Relay amplification factor has become the basis for most AF relaying network models. Therefore, we consider the linear connection system model as illustrated in Fig. 1 in which the source (S) is assumed fixed but both the relay (R) and destination (D) can move. In this model, we assume independent Rayleigh fading for the channel gains $h_{S R} \sim C N\left(0, \sigma_{S R}^{2}\right)$, $h_{R D} \sim C N\left(0, \sigma_{R D}^{2}\right)$ and $\mathrm{h}_{\mathrm{SD}} \sim \mathrm{CN}\left(0, \sigma_{\mathrm{RD}}^{2}\right)$. We also assume receiver noise $z_{S R} \sim C N\left(0, N_{0}\right), \quad z_{R D} \sim C N\left(0, N_{o}\right) \quad$ and $z_{S D} \sim C N\left(0, N_{0}\right)$. It is assumed that all node inputs experience identical additive white Gaussians noise (AWGN), therefore, $Z_{S D}=Z_{S R}=Z_{R D}=n_{D}$. The term $C N(.,$.$) represents the complex Gaussian random variable$ with the first and the second parameter denoting the mean and the variance, respectively. The relay and source transmits powers are denoted by $P_{\mathrm{R}}$ and $P_{\mathrm{S}}$, respectively. Therefore, the total transmits power in the cell, $P_{\mathrm{T}}=P_{\mathrm{R}}+P_{\mathrm{S}}$.

To facilitate transmission, a Time-Division-MultipleAccess (TDMA) subcarrier allocation with two time slots is considered. As described in Table 1, the relay node simply amplifies the received signal and then forwards it to the destination.

In Half-Duplex (HD) mode, the AF relay takes two time slots to transmit a packet from source to destination. In the first timeslot, the source broadcasts its unit-energy signal $x$ and the signal received by destination is:

$$
y_{S D}=\sqrt{P_{S}}\left|h_{S D}\right|^{2} x+n_{D}
$$

The signal received by relay is:

$$
y_{S R}=\sqrt{P_{S}}\left|h_{S R}\right|^{2} x+n_{D}
$$

Table 1. TDMA transmission protocol for the HD relaying system

\begin{tabular}{clll}
\hline & BS & RS & MS \\
\hline Timeslot 1 & Transmits & Listens & Listens \\
Timeslot 2 & - & Transmits & Listens \\
\hline
\end{tabular}

In the second timeslot, the relay multiplies the received signal by a relay amplification factor (relay gain) $D^{2}$ and then forwards the amplified signal to destination. Then the signal at destination through relay can be expressed by:

$$
\begin{aligned}
& y_{R D}=\sqrt{P_{R}}\left|h_{R D}\right|^{2} y_{S R} D^{2}+n_{D} \\
& =\sqrt{P_{R}}\left|h_{R D}\right|^{2} \sqrt{P_{S}}\left|h_{S R}\right|^{2} x D^{2}+\sqrt{P_{R}}\left|h_{R D}\right|^{2} n_{D} D^{2}+n_{D}
\end{aligned}
$$

The effective noise term, $\breve{n}_{D}$ is equivalent to the noise variance $N_{0}$ and it can be derived from the second and third terms of Equation 3 as:

$$
N_{0}=n_{D}\left(1+\sqrt{P_{R}}\left|h_{R D}\right|^{2} D^{2}\right)
$$

The relay amplification factor $D^{2}$ as seen in the first term of Equation 3 is expected to provide proper amplification to the relay while equalizing the effect of the channel fading and prevents the relay gain from saturating when the S-R channel undergoes deep fade. However, this gain also amplifies the effective noise term in Equation 4, by a margin which depends on the choices of the scaling parameters.

\section{Proposed AF Amplification Factor}

Traditional amplification factor can be expressed by:

$$
D^{2}=\frac{\rho}{P_{S} \sigma_{S R}^{2}+N_{0}}
$$

where, the constant $\rho$ has been set at $\rho=1$ or $\rho=P_{R}$. The only parameter of the relay gain in Equation 5 that can change is the variance, $\sigma_{S R}^{2}$, therefore it has only one degree of freedom. To further reduce the combined effects of fading and noise, the value of $\rho$ must be chosen to give the amplification factor another degree of freedom; one in the denominator and another in the numerator of $D^{2}$. Therefore, we propose an amplification factor expressed by:

$$
D^{2}=\frac{\alpha^{2}}{P_{s} \sigma_{S R}^{2}+N_{0}}
$$

where, $\alpha^{2}=\beta(1-d)^{-k}$ is a non-fading variable path gain, $\beta$ $=1$ is the path-loss exponent of a reference distance $d_{0}, d$ is the normalized relay distance from the source given by $d=\frac{d^{\text {effec }}}{d_{0}}$ and $k$ is the path-loss exponent of the S-R 
channel. The effective distance, $d^{\text {effec }}$, is the actual distance of $\mathrm{R}$ and $\mathrm{D}$ in meters with respect to the location of $\mathrm{S}$, and $d_{0}$ is taken to be the radius of the cell. Therefore, if the channel state information of the S-D channel coefficient $\left|h_{S R}\right|^{2}$ can be estimated, the proposed scheme can also perform variable-gain relaying, otherwise double-semi-blind AF relaying will be provided. The instantaneous and average signal-to-noise ratio (SNR) of S-D, S-R and R-D channel gains are defined, respectively, as:

$$
\begin{gathered}
Y_{S R}=\frac{P_{S}\left|h_{S R}\right|^{2}}{n_{D}}, Y_{S D}=\frac{P_{S}\left|h_{S D}\right|^{2}}{n_{D}}, Y_{R D}=\frac{P_{R}\left|h_{S R}\right|^{2}}{n_{D}} \\
\overline{\mathrm{Y}}_{S R}=\frac{P_{S} \sigma_{S R}^{2}}{N_{o}}, \overline{\mathrm{Y}}_{S D}=\frac{P_{S} \sigma_{S D}^{2}}{N_{o}}, \overline{\mathrm{Y}}_{R D}=\frac{P_{R} \sigma_{R D}^{2}}{N_{o}}
\end{gathered}
$$

The following section will compute the instantaneous SNR, average capacity and outage probability for the proposed and some current AF schemes.

\section{Instantaneous SNR}

In cooperative relaying communication, the Maximum Ratio Combiner (MRC) can be used to combine signals on the S-D and S-R-D links. Therefore, the combined signal at destination through the $\mathrm{AF}$ relaying can be written as:

$$
\begin{aligned}
& y_{A F}=\sqrt{P_{S}}\left|h_{S D}\right|^{2} x+n_{D}+\sqrt{P_{R}}\left|h_{R D}\right|^{2} \sqrt{P_{S}}\left|h_{S R}\right|^{2} x D^{2} \\
& +\sqrt{P_{R}}\left|h_{R D}\right|^{2} n_{D} D^{2}+n_{D}
\end{aligned}
$$

The instantaneous SNR for the proposed AF scheme can be expressed by:

$$
\mathrm{Y}_{A F_{i}}=\mathrm{Y}_{S D}+\frac{\mathrm{Y}_{S R} \mathrm{Y}_{R D}}{\mathrm{Y}_{R D}+\frac{1}{N_{0} D_{i}^{2}}}
$$

where, $D_{i}^{2}, \mathrm{i}=1, \ldots \mathrm{p}$ is the corresponding amplification factor for the AF schemes defined, respectively, as:

$$
\begin{aligned}
& D_{1}^{2}=\frac{P_{R}}{P_{S} \sigma_{S R}^{2}+N_{0}} \\
& D_{2}^{2}=\frac{1}{P_{S} \sigma_{S R}^{2}+N_{0}} \\
& D_{p}^{2}=\frac{\alpha^{2}}{P_{S} \sigma_{S R}^{2}+N_{0}}
\end{aligned}
$$

The $D_{1}^{2}, D_{2}^{2}$ and $D_{p}^{2}$ are the amplification factors for AF relaying schemes denoted, respectively, as AF1, AF2 and proposed AF.

\section{Outage Probability}

We follow the same procedure as used in (Rasouli, 2012), to compute the outage probability for the different AF schemes. The Probability Density Function (PDF) of SNR of the direct link (S-D) in AF relaying can be modeled by an exponential random variable with parameter $\beta_{d}=\frac{1}{\overline{\mathrm{Y}}_{S D}}$. We model the pdf of the SNRs of indirect link approximated by an exponential random variable with parameter, $\beta_{i}=\frac{1}{\overline{\mathrm{Y}}_{S R}}+\frac{1}{\overline{\mathrm{Y}}_{R D}}$. Using the moment generating function approach, the pdf of $Y_{A F}$, $f_{\tau A F}\left(Y_{A F}\right)$, can be written as:

$$
f_{\tau_{A F}}\left(\mathrm{Y}_{A F}\right)=\frac{\beta_{d} \beta_{i}\left(e^{-\beta_{i} \mathrm{Y}_{A F}}-e^{-\beta_{d} \mathrm{Y}_{A F}}\right)}{\beta_{d}-\beta_{i}}
$$

Generally, the probability of outage for AF relaying for an independent Rayleigh fading channels is, therefore, calculated as:

$$
\begin{aligned}
& P_{\text {out }}^{A F}=\operatorname{Prob}\left[\mathrm{Y}_{A F}<\mathrm{Y}^{\text {th }}\right]=\int_{0}^{\mathrm{Y}^{\text {th }}} f_{\tau_{A F}}\left(\mathrm{Y}_{A F}\right) d \mathrm{Y}_{A F} \\
& =\frac{\beta_{i}}{\beta_{d}-\beta_{i}}\left(e^{-\beta_{d} \mathrm{Y}^{\text {th }}}-1\right)-\frac{\beta_{d}}{\beta_{d}-\beta_{i}}\left(e^{-\beta_{i} \mathrm{Y}^{\text {th }}}-1\right)
\end{aligned}
$$

Because $\alpha^{2}$ is non-fading path gain, its average power gain $\bar{\alpha}^{2}=\alpha^{2}$ is assumed for the proposed $\mathrm{AF}$ amplification factor in Equation 13 and so its outage probability can be derived as:

$$
\begin{aligned}
& P_{\text {out }}^{\text {proposed }}= \frac{\frac{1}{\overline{\mathrm{Y}}_{S R}}+\frac{1}{\overline{\mathrm{Y}}_{R D} \alpha^{2}}}{\overline{\overline{\mathrm{Y}}}_{S D}}-\frac{1}{\overline{\mathrm{Y}}_{S R}}-\frac{1}{\overline{\mathrm{Y}}_{R D} \alpha^{2}}\left(e^{\frac{-\mathrm{Y}^{\text {th }}}{\overline{\mathrm{Y}}_{S D}}}-1\right) \\
&-\frac{1}{\overline{\overline{\mathrm{Y}}}_{S D}}\left(e^{-\mathrm{Y}^{\text {th }}\left(\frac{1}{\overline{\mathrm{Y}}_{S R}}+\frac{1}{\overline{\mathrm{Y}}_{R D} \alpha^{2}}\right)}-1\right) \\
& \frac{1}{\overline{\mathrm{Y}}_{S D}}-\frac{1}{\overline{\mathrm{Y}}_{S R}}-\frac{1}{\overline{\mathrm{Y}}_{R D} \alpha^{2}}()
\end{aligned}
$$

For the AF amplification factors in Equation 11 and 12, respectively, the outage probability for AF1 and AF2 schemes are derived from Equation 15 as follow:

$$
\begin{aligned}
& P_{\text {out }}^{A F 1}=\frac{\frac{1}{\overline{\mathrm{Y}}_{S R}}+\frac{1}{\overline{\mathrm{Y}}_{R D} P_{R}}}{\frac{1}{\overline{\mathrm{Y}}_{S D}}-\frac{1}{\overline{\mathrm{Y}}_{S R}}-\frac{1}{\overline{\mathrm{Y}}_{R D} P_{R}}}\left(e^{\frac{-\mathrm{Y}^{\text {th }}}{\overline{\mathrm{S}}_{S D}}}-1\right) \\
& -\frac{\frac{1}{\overline{\mathrm{Y}}_{S D}}}{\frac{1}{\overline{\mathrm{Y}}_{S D}}-\frac{1}{\overline{\mathrm{Y}}_{S R}}-\frac{1}{\overline{\mathrm{Y}}_{R D} P_{R}}}\left(e^{-\mathrm{Y}^{\text {th }}\left(\frac{1}{\overline{\mathrm{Y}}_{S R}}+\frac{1}{\overline{\mathrm{Y}}_{R D} P_{R}}\right)}-1\right)
\end{aligned}
$$




$$
\begin{gathered}
P_{\text {out }}^{A F 2}=\frac{\frac{1}{\overline{\mathrm{Y}}_{S R}}+\frac{1}{\overline{\mathrm{Y}}_{R D}}}{\frac{1}{\overline{\mathrm{Y}}_{S D}}-\frac{1}{\overline{\mathrm{Y}}_{S R}}-\frac{1}{\overline{\mathrm{Y}}_{R D}}}\left(e^{\frac{-\mathrm{Y}^{\text {th }}}{\overline{\mathrm{S}}_{S D}}}-1\right) \\
-\frac{\frac{1}{\overline{\mathrm{Y}}_{S D}}}{\frac{1}{\overline{\mathrm{Y}}_{S D}}-\frac{1}{\overline{\mathrm{Y}}_{S R}}-\frac{1}{\overline{\mathrm{Y}}_{R D}}}\left(e^{-\mathrm{Y}^{\text {th }}\left(\frac{1}{\overline{\mathrm{Y}}_{S R}}+\frac{1}{\overline{\mathrm{Y}}_{R D}}\right)}-1\right)
\end{gathered}
$$

\section{Average Capacity}

The Shannon maximum error-free capacity formula in AWGN channel is $C=B \log _{2}\left(1+\gamma_{t}\right)$, where $\gamma_{t}$ is SNR. For AF relaying schemes at the destination terminal, the instantaneous capacity can be expressed by:

$$
C^{A F_{i}}=\frac{B}{2} \log _{2}\left(1+\mathrm{Y}_{S D}+\frac{\mathrm{Y}_{S R} \mathrm{Y}_{R D}}{\mathrm{Y}_{R D}+\frac{1}{N_{0} D_{i}^{2}}}\right)
$$

where, $B$ is system bandwidth and the division by 2 indicates that HD transmission protocol takes two timeslots for each symbol and $C^{A F i}$ is the capacity corresponding to AF1, AF2 and proposed AF schemes using $D_{i}^{2}$, respectively, as defined in Equation 11-13. However, the average capacity can be calculated by taking the average of Equation 19 over a number of iterations.

\section{Numerical Results}

In the linear connection model of Fig. 1, the channel power gains are set at $\sigma_{S D}^{2}=1, \quad \sigma_{S R}^{2}=d^{-3}$ and $\sigma_{R D}^{2}=(1-d)^{-3}$ where $\mathrm{d}$ is the normalized relay distance from the source and the path-loss exponent is 3 . Therefore, $\alpha^{2}=(1-d)^{-3}$ in the proposed AF scheme. The destination is assumed to be on the edge of the cell at normalized distance of $\mathrm{d}=1$ and the noise power is set at $N_{0}=1 \mathrm{~mW}$. We assume an average SNR threshold, $Y^{t h}=$ $5 d B$ to compute the outage probability.

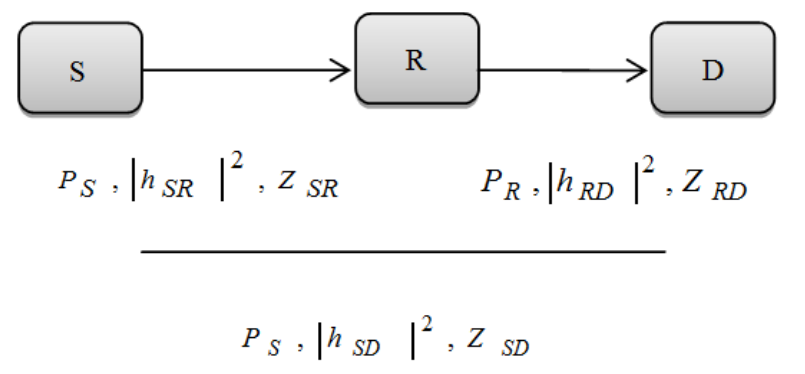

Fig. 1. The relay transmission: Linear connection model
Average Capacity and Outage Probability as a function Transmit Power

In Fig. 2, the proposed AF scheme is shown to achieve the highest average capacity, followed by AF2 and $\mathrm{AF} 1$ schemes when the total transmit power, $\mathrm{P}_{\mathrm{T}}$, is equally allocated between the source and the relay. It is evident that the proposed scheme achieves the highest capacity because it uses additional variable parameter which increases its average SNR. The AF1 achieves the lowest capacity because it uses the relay power, $\mathrm{P}_{\mathrm{R}}$, as a fixed scaling parameter which tends to amplify the noise more compared to AF2 which uses a unity value. As shown in Fig. 3, when the relay power is increased to two-third of the total transmits power, the average capacity of the proposed AF at each total transmits power is increased compared to that in Fig. 2. For AF1, the increase occurs only at $\mathrm{P}_{\mathrm{T}}$ below $25 \mathrm{dBm}$ while AF2 is almost unaffected because of its unity scaling parameter.

In Fig. 4, the proposed AF is depicted as achieving the lowest outage probability; implying that it is able to provide the highest SNR. The AF1 is seen to produce the worst probability of outage which only improves as the total transmits power increases. Therefore, for AF1 to achieve comparable outage probability as AF2 the total transmits power must be increased beyond $30 \mathrm{dBm}$; suggesting that it is not power-efficient.

However, when two-thirds of the total transmits power is allocated to the relay; the outage probability of the proposed AF reduces further while that of AF1 appears to be worse at high transmits powers as shown in Fig. 5. The figure also shows that, although, AF2 achieves very little improvement, the difference between it and that of proposed AF widens with increase in the share of the relay power.

\section{Average Capacity and Outage Probability as a Function Relay Distance}

In this section, we assume a total transmit power, $\mathrm{P}_{\mathrm{T}}$ $=20 \mathrm{dBm}$, which can be distributed either equally or unequally between the relay and source. As shown in Fig. 6, the proposed AF achieves the highest average capacity compared to other schemes at most of the relay distances. The proposed AF is also seen to attain its maximum value at a farther distance from the destination compared to AF2.

It is evident that AF1 amplifies the greatest amount of noise; hence its average capacity is the poorest at all relay distances. However, it can be noticed that as the relay moves closer, the SNR and hence the average capacity of AF2 and proposed AF reduce since the channel gains which are distance-dependent are then expected to be higher. 


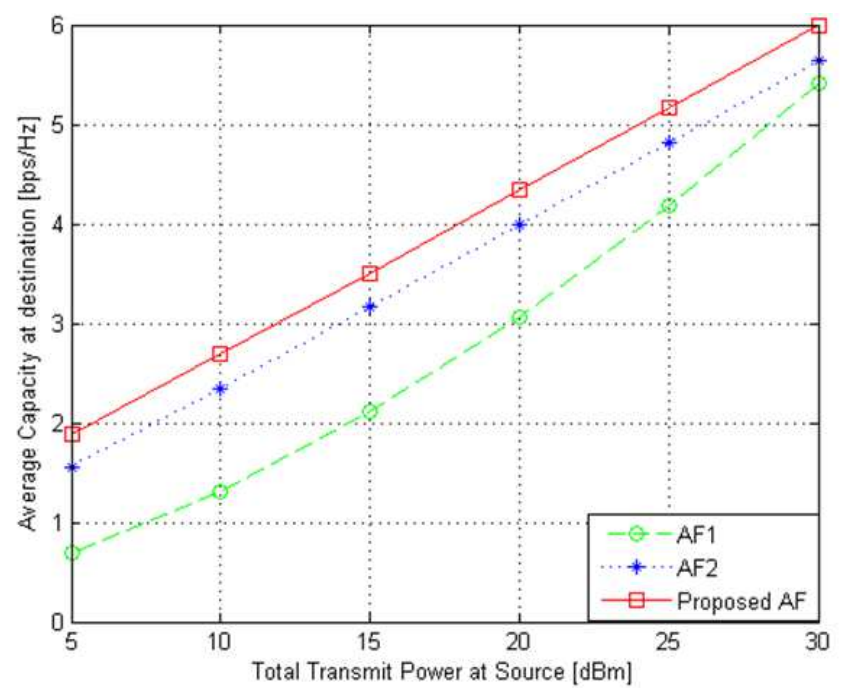

Fig. 2. Average capacity as a function of total transmit power at $\mathrm{P}_{\mathrm{R}}=0.5 \mathrm{P}_{\mathrm{T}}, \mathrm{P}_{\mathrm{S}}=0.5 \mathrm{P}_{\mathrm{T}}$

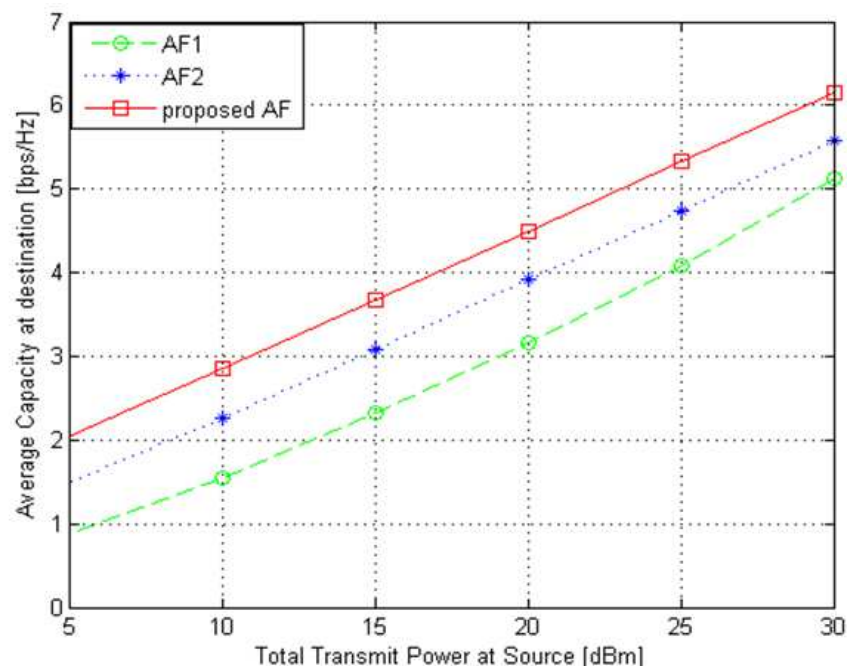

Fig. 3. Average capacity as a function of total transmit power at $\mathrm{P}_{\mathrm{R}}=0.75 \mathrm{P}_{\mathrm{T}}, \mathrm{P}_{\mathrm{S}}=0.25 \mathrm{P}_{\mathrm{T}}$

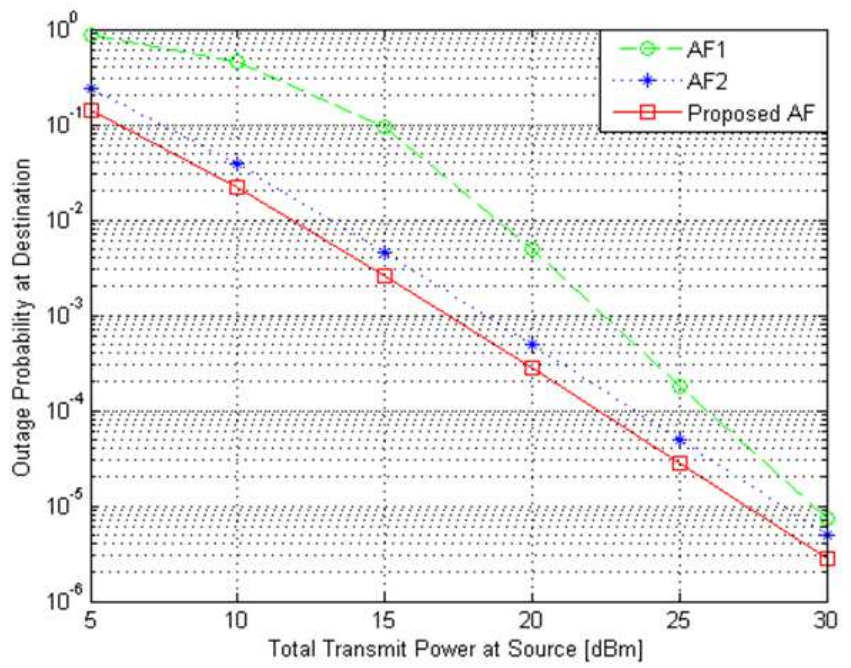

Fig. 4. Outage probability as a function of total transmit power at $\mathrm{P}_{\mathrm{R}}=0.5 \mathrm{P}_{\mathrm{T}}, \mathrm{P}_{\mathrm{S}}=0.5 \mathrm{P}_{\mathrm{T}}$ 


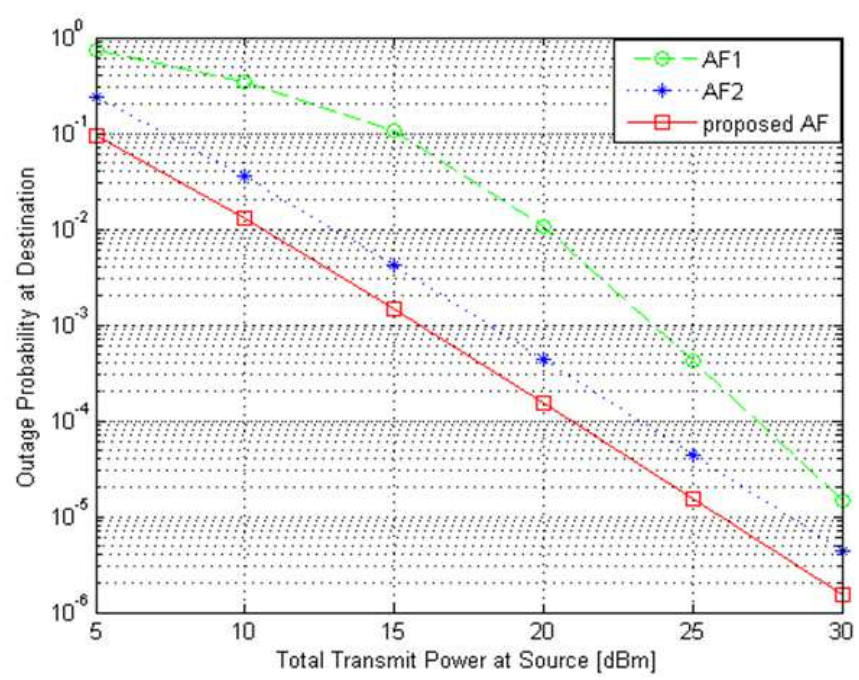

Fig. 5. Outage probability as a function of total transmit power at $P_{R}=0.75 \mathrm{P}_{T}, \mathrm{P}_{\mathrm{S}}=0.25 \mathrm{P}_{T}$

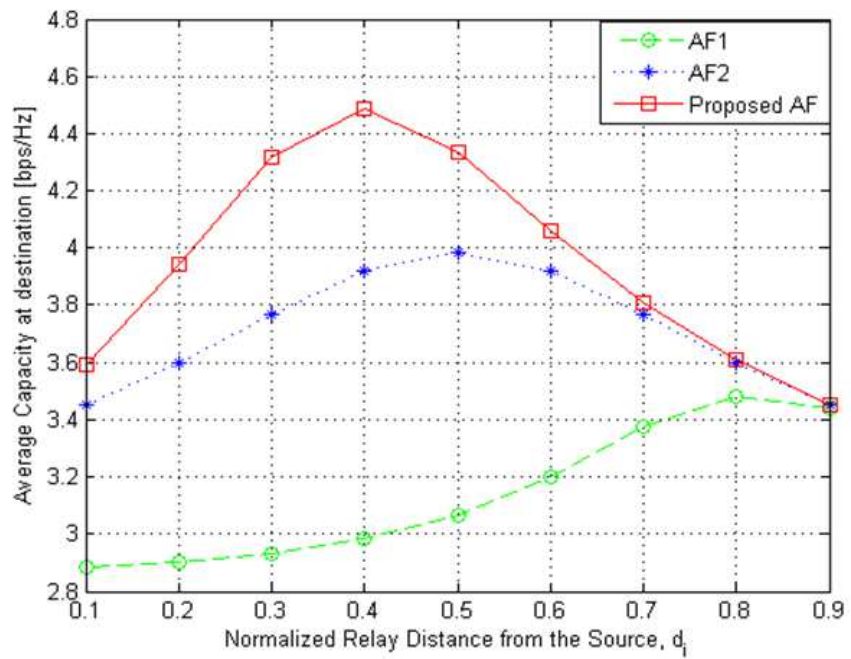

Fig. 6. Average capacity as a function of normalized relay distance, $d$, from source at $P_{R}=0.5 P_{T}, P_{S}=0.5 P_{T}$

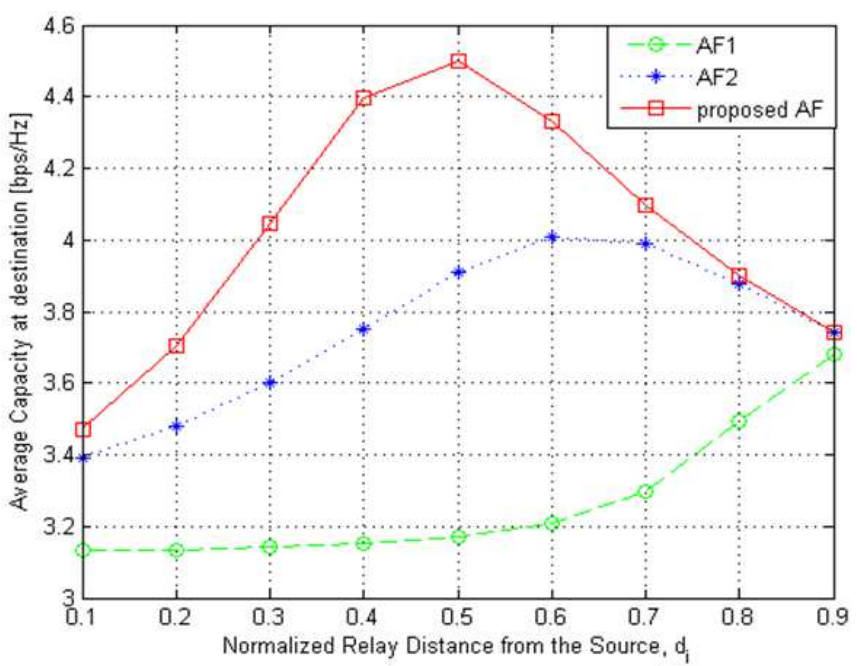

Fig. 7. Average capacity as a function of normalized relay distance, $d$, from source at $P_{R}=0.75 P_{T}, P_{S}=0.25 P_{T}$ 


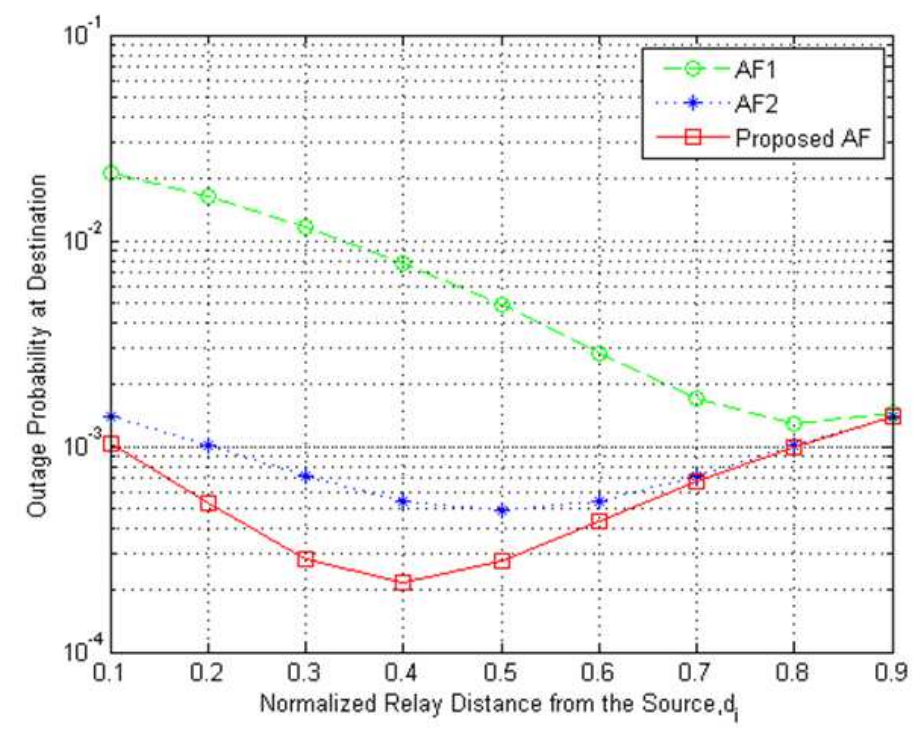

Fig. 8. Outage probability as a function of normalized relay distance, $d$, from source at $P_{R}=0.5 P_{T}, P_{S}=0.5 P_{T}$

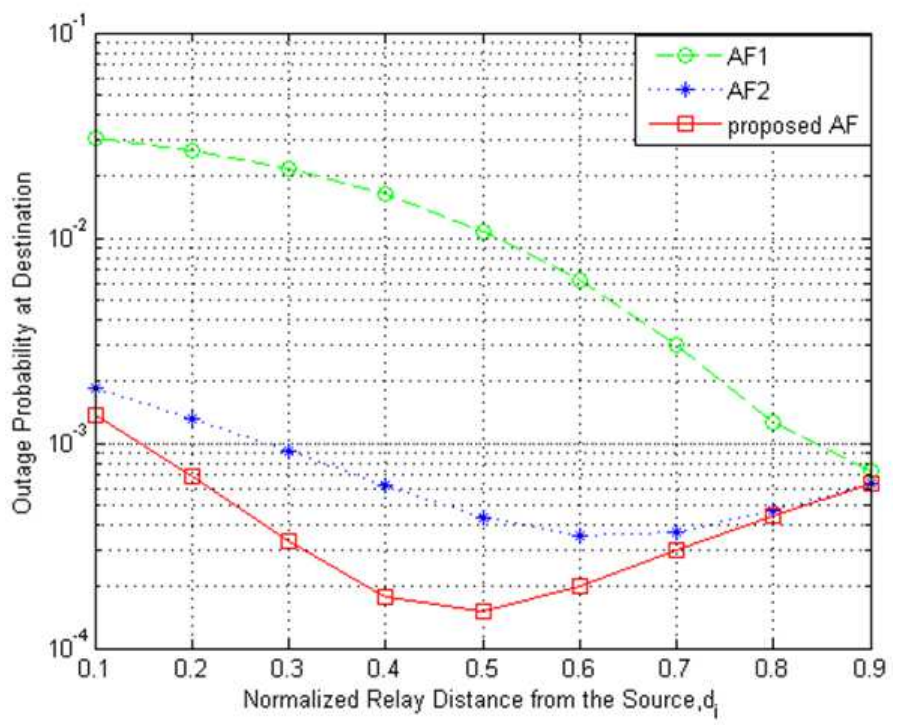

Fig. 9. Outage probability as a function of normalized relay distance, $d$, from source at $P_{R}=0.75 P_{T}, P_{S}=0.25 P_{T}$

In Fig. 7 when the relay power share is increased to two-third, the maximum average capacity values of AF1, AF2 and proposed AF shift to $\mathrm{d}=0.9, \mathrm{~d}=0.6$ and $\mathrm{d}=$ 0.5 , respectively; a shift to right by 1 unit of normalized distance, d, when viewed against Fig. 6. The superiority of the proposed AF is seen to be maintained, however, it can be observed that the average capacities before the optimal relay locations (location where maximum value occurs) are reduced while those after are increased for all the schemes.

In Fig. 8, the AF1 and AF2 are outperformed by the proposed AF scheme which achieves its minimum outage probability at $d=0.4$. It can also be seen that AF 1 is the most unreliable, especially at closer distances of the relay to the source. However, when the relay power is increased by one-third to the decrease in power of the source by the same margin; Fig. 9 shows that the probability of outage before and after the optimal relay location is reduced for all the relaying schemes except AF1; because the use of relay power as a scaling constant further worsens its performance.

\section{Conclusion}

In this study, we have analyzed some AF amplification factors and noted that their main problem is that they rely on only one variable parameter to 
perform the amplification. Hence, they have only one degree of freedom and this limits their abilities to effectively deal with the combined effect of channel fading and noise. We therefore, propose a new $\mathrm{AF}$ amplification factor which provides additional degree of freedom through the forward channel. Numerical results show that the proposed AF scheme outperforms the current ones in terms of average capacity and outage probability both as a function of total transmit power and relay distance. This means that the proposed AF scheme can bring statistical performances of $\mathrm{AF}$ closer to that of DF relaying, which hitherto outperforms it. The proposed scheme can even provide a more attractive practical solution, because AF is generally considered better in terms of simplicity of implementation. The results also show that by increasing or decreasing the power share of the relay, the optimal location of the relay is not only moved closer to or farther away from the destination, the total average capacity at that location is also correspondingly increased or decreased.

\section{Acknowledgement}

This work is supported by DPI and in part by Zamallah Graduate Scholarship of the University of Malaysia, Sarawak.

\section{Funding Information}

The authors of this paper have no financial funding to report.

\section{Author's Contributions}

Olalekan Bello: Undertakes the analysis and interpretation of results. Also works in writing the manuscript.

Hushairi Zen: Design the research and prepare the workflow. Also works in writing the manuscript.

AL-Khalid Othman: Studied the related works and tested the models. Also works in writing the manuscript.

Khairuddin Abdul Hamid: Improved the methodological frameworks and re-formatted the presentation. Also works in writing the manuscript.

\section{Ethics}

This article is original and contains unpublished materials. The corresponding author confirms that all of the other authors have read and approved the manuscript and no ethical issues involved.

\section{References}

Alvi, S.H. and S. Wyne, 2014. On amplify-and-forward relaying over Hyper-Rayleigh fading channels. Radio Eng., 23: 1226-1233.
Anghel, P. and M. Kaveh, 2003. Multi-user space-time coding in cooperative networks. Proceedings of the IEEE International Conference on Acoustics, Speech and Signal Processing, Apr. 6-10, IEEE Xplore Press, pp: 73-76.

DOI: 10.1109/ICASSP.2003.1202544

Berger, S. and A. Wittneben, 2005. Cooperative distributed multiuser MMSE relaying in wireless adhoc networks. Proceedings of the Asilomar Conference on Signals, Systems and Computers, Oct. 28-Nov. 1, IEEE Xplore Press, Pacific Grove, CA, pp: 1072-1076.

DOI: 10.1109/ACSSC.2005.1599923

Cheng, W. and T. Chen, 2015. Dual-hop fixed gain relaying transmissions with semi-blind in asymmetric multipath/shadowing fading channels. Open Electrical Electronic Eng. J., 9: 82-90.

DOI: $10.2174 / 1874129001509010082$

Cheng, W. and Y. Huang, 2015. Ergodic capacity of dual-hop semi-blind AF relaying over composite fading channels using mixture gamma distribution. WSEAS Trans. Commun., 14: 11-16.

Hasna, M.O. and M.S. Alouini, 2004. A performance study of dual-hop transmissions with fixed gain relays. IEEE Trans. Wirel. Commun., 3: 1963-1968. DOI: 10.1109/TWC.2004.837470

Laneman, J.N., D.N.C. Tse and G.W. Wornell, 2004. Cooperative diversity in wireless networks: Efficient protocols and outage behavior. IEEE Trans. Inform. Theory, 50: 3062-3080. DOI: 10.1109/TIT.2004.838089

Liu, K.J.R., A.K. Sadek, W. Su and A. Kwasinski, 2008. Cooperative Communications and Networking. 1st Edn., Cambridge University Press, ISBN-10: 9780511754524

Nabar, R., H. Bolcskei and F. Kneubuhler, 2004. Fading relay channels: Performance limits and space-time signal design. IEEE J. Selected Areas Commun., 22: 1099-1109. DOI: 10.1109/JSAC.2004.830922

Osamah, S.B. and K. Michel, 2012. Performance analysis of dual-hop systems with fixed-gain relays over generalized $\eta-\mu$ fading channels. Proceedings of the IEEE Global Communications Conference, Dec. 3-7, IEEE Xplore Press, Anaheim, CA, pp: 4148-4152. DOI: 10.1109/GLOCOM.2012.6503767

Peppas, K., A. Mansour and G.T. Tombras, 2010. Dualhop transmissions with fixed-gain relays over Generalized-Gamma fading channels. J. Telecommun., 1: 87-93.

Rasouli, H., 2012. Cooperative subcarrier and power allocation in OFDM based relaying system. Unpublished Dissertation in partial fulfillment of the requirements for the degree of Doctor of Philosophy, Ryerson University, Canada. 
Saleh, A., S. Redana, B. Raaf, T. Riiihonen and J. Hamalainen et al., 2009. Performance of amplifyand-forward and decode-and-forward relays in LTEadvanced. Proceedings of the IEEE 70th Vehicular Technology Conference Fall, Sept. 20-23, IEEE Xplore Press, Anchorage, AK, pp: 1-5.

DOI: 10.1109/VETECF.2009.5378824

Souryal, M.R., 2006. Performance of amplify-andforward and decode-and-forward relaying in Rayleigh fading with turbo codes. Proceedings of the IEEE International Conference on Acoustics, Speech and Signal Processing, May 14-19, IEEE Xplore Press, Toulouse, pp: 681-684.

DOI: 10.1109/ICASSP.2006.1661060
Xia, M., C. Xing, Y.C. Wu and S. Aïssa, 2011. Exact performance analysis of Dual-hop semi-blind AF relaying over arbitrary Nakagami-m fading channels. IEEE Trans. Wireless Commun., 10: 3449-3459. DOI: 10.1109/TWC.2011.080311.102267 